\title{
Measurement of Isolated Photon Production in Deep Inelastic Scattering at HERA
}

\author{
Katharina Müller \\ University of Zurich \\ Winterthurerstrasse 190 , CH - 8057 Zuerich - Switzerland
}

\begin{abstract}
Results are presented on the production of isolated photons $e p \rightarrow e+\gamma+X$ in deep inelastic scattering with a four-momentum transfer squared of $4<Q^{2}<150 \mathrm{GeV}^{2}$, inelasticity $y>0.05$ and a mass of the hadronic system $W_{X}>50 \mathrm{GeV}$. Isolated photons are selected in the range $3<E_{T}^{\gamma}<10 \mathrm{GeV}$ and pseudorapidity range $-1.2<\eta^{\gamma}<1.8$. The cross sections, both inclusive and for events with zero or at least one additional jet, are measured with the $\mathrm{H} 1$ detector at the electron-proton collider HERA. The analysis is based on a total integrated luminosity of $227 \mathrm{pb}^{-1}$. The measurements are compared to MC predictions and to a LO calculation. Both approaches underestimate the observed signal, particularly in the low $Q^{2}$ domain.
\end{abstract}

\section{Introduction}

Photons originating from the hard interaction in high energy collisions are a sensitive probe for precision tests of perturbative QCD and provide information on the proton structure. In contrast to measurements using hadrons, a measurement with isolated photons minimises uncertainties from parton fragmentation, hadronisation or jet identification. Furthermore, the experimental uncertainties of the energy measurement are smaller for electromagnetic showers in the calorimeter initiated by photons than for jets of hadrons. The measurement of isolated photons in deep-inelastic scattering (DIS) provides a test of QCD in a kinematic range with two hard scales, the four-momentum transfer squared $Q^{2}$ of the exchanged virtual photon and the transverse energy of the emitted photon.

Previously H1 and ZEUS $[2,3,4]$ have measured prompt photon cross sections in photoproduction. An analysis of the isolated photon cross section in DIS with $Q^{2}$ larger than $35 \mathrm{GeV}^{2}$ has been published by ZEUS [5].

In DIS the final state photon is emitted by a quark ( $Q Q$ subprocess), by wide angle radiation from the lepton ( $L L$ subprocess) or by interference ( $L Q$ subprocess). Since the photon and the scattered electron are well separated in the present analysis, low angle QED radiation is suppressed. The $L L$ and the more interesting $Q Q$ process can not be distinguished experimentally on event basis, but only by comparison with predictions.

This paper presents the measurement of isolated photon production $e+p \rightarrow e+\gamma+X$, where $X$ is anything. Jet production in events with isolated photons and no additional jet or with at least one jet is also investigated. The measurement range covered by this analysis significantly extends the kinematic range probed by the previous measurement [5]. The results are compared to a recent leading order, $\mathcal{O}\left(\alpha^{3} \alpha_{s}^{0}\right)$, calculation $[6,7]$ and to the predictions of the event generators PYTHIA [8], simulating the $Q Q$ process, and RAPGAP [9] for the $L L$ process. 


\section{Analysis method}

\section{$2.1 \quad$ Event selection}

The event sample used in this analysis was collected with the H1 Detector [10, 11] at HERA in the period from 1999 to 2005 at a centre-of-mass energy of $318 \mathrm{GeV}$. The corresponding integrated luminosity is $227 \mathrm{pb}^{-1}$.

DIS events are selected with the scattered electron identified by a compact electromagnetic cluster with an energy $E_{e}^{\prime}>10 \mathrm{GeV}$ and a polar angle $\theta_{e}<177^{\circ}$ in the backward calorimeter (SpaCal). $Q^{2}$ is restricted to $4<Q^{2}<150 \mathrm{GeV}^{2}$ and the inelasticity to $y>0.05$. A photon candidate is identified as a cluster in the electromagnetic section of the liquid argon (LAr) calorimeter $[12,13]$ with a transverse energy $3<E_{T}^{\gamma}<10 \mathrm{GeV}$ and pseudo-rapidity $-1.2<\eta^{\gamma}<1.8$ and no track pointing to it within $20 \mathrm{~cm}$. Background from elastic Compton scattering is suppressed by a cut on the invariant mass of the hadronic system, $W_{X}>50 \mathrm{GeV}$. Jets with a transverse momentum of $P_{T}^{\text {jet }}>2.5 \mathrm{GeV}$ and a pseudorapidity in the range $-2.0<\eta^{j e t}<2.1$ are reconstructed using the $k_{T}$ algorithm [14], where jets are treated as massless. The algorithm is used with a $P_{T}$-weighted recombination scheme and the separation parameter $R_{0}$ is set to one. The jet containing the photon candidate is classified as photon-jet the others as hadronic jets. Hadronic jets are restricted to the pseudorapidity range $-1.0<\eta^{j e t}<2.1$. To ensure isolation of the photon, the fraction of the transverse energy of the photon-jet carried by the photon candidate has to be larger than $90 \%$. The isolation criterion rejects background from photons induced by hadron decays.

\subsection{Photon signal extraction}

The isolated photon signal is extracted using a shower shape analysis. It is based on the fact that electromagnetic showers in the calorimeter initiated by isolated photons tend to be more narrow, symmetric and compact and tend to start off slightly deeper in the calorimeter than background showers. Six different shower shape variables are defined. The background originates mainly from neutral hadrons with subsequent decays to multi-photon states. Probability density functions are determined for the six shower shape variables, using simulated single particles events with photons and a mix $\left(\pi^{0}, \eta, \eta^{\prime}, \rho, \omega, K^{\star}, K_{L}^{0}, K_{S}^{0}, n\right.$ and $\left.\bar{n}\right)$ of neutral hadrons, respectively. The relative contribution of any neutral hadron is taken from the RAPGAP MC. The multi-dimensional photon and background probability densities are taken as the product of the respective shower shape densities. For each measured event a discriminator is formed. It is defined as the photon probability density divided by the sum of the probability densities for photons and background. The discriminator produces generally larger values for isolated photons than for the multi-photon clusters. The contribution of photons and neutral hadrons in any analysis bin is determined by independent minimum- $\chi^{2}$ fits of the signal and background discriminator distributions to the data distribution.

\section{Isolated photon cross section}

Differential cross sections $d \sigma / d \eta^{\gamma}$ and $d \sigma / d Q^{2}$ for the inclusive isolated photon cross section are shown in Figure 1 together with the predictions by the LO calculation. The uncertainty on the shower description gives the dominant contribution to the systematical error. The calculation is corrected to hadron level. The corrections amount to at most $-30 \%$. 
The LO calculation underestimates the cross sections in average by roughly $44 \%$, most significantly at low $Q^{2}$. The relative contribution of $L L$ and $Q Q$ depends strongly on $\eta^{\gamma}$ and $Q^{2}$. At high and medium $\eta^{\gamma}$ and low $Q^{2}$, radiation by the quark dominates. The data are also compared to the MC prediction of PYTHIA for the radiation from the quark and RAPGAP for the radiation from the electron (not shown). The sum of the MC predictions gives a very similar result as the LO calculation and also underestimates the data significantly. The Inclusive isolated photon production
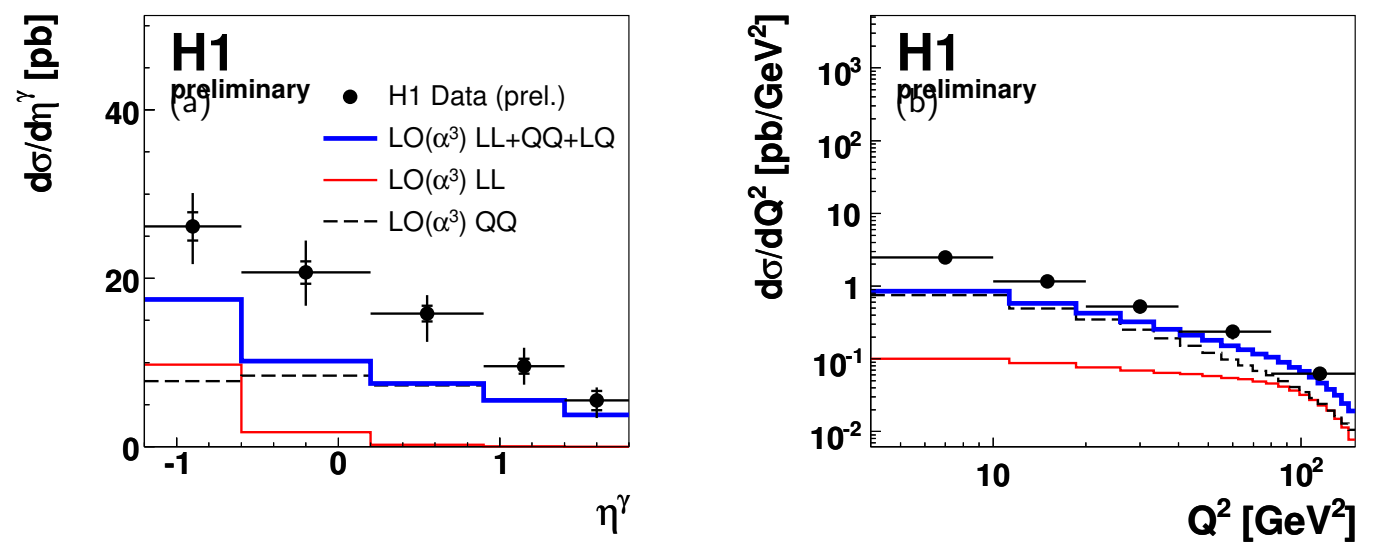

Figure 1: Inclusive differential isolated photon cross sections $d \sigma / d \eta^{\gamma}$ (a), $d \sigma / d Q^{2}$ (b) for $3<E_{T}^{\gamma}<10 \mathrm{GeV},-1.2<\eta^{\gamma}<1.8, W_{X}^{2}>2500 \mathrm{GeV}^{2}$ and $4<Q^{2}<150 \mathrm{GeV}^{2}$. The inner error bars on the data points indicate the statistical error, the full error bars contain in addition the systematic errors added in quadrature. The cross sections are shown together with a leading order, $\alpha^{3} \alpha_{s}^{0}$, calculation corrected for hadronisation effects, $L L$ corresponding to radiation from the electron and $Q Q$ to radiation from the quark.

cross section for jet production in association with isolated photons is studied using events with no or at least one hadronic jet. Figure 2 shows the differential cross sections $d \sigma / d \eta^{\gamma}$ for isolated photons with no-jet (a) and with jets (b). For both samples the LO calculation describes reasonably well the shape but is too low in normalisation. The $L L$ contribution is largely suppressed for the photon plus no-jet sample due to the cut on $W_{X}$. The cross section for photon plus jets production is roughly two times higher than for the photon plus no-jets production.

\section{Conclusions}

The cross section for isolated photon production in deep inelastic scattering has been measured in the squared momentum transfer range $4<Q^{2}<150 \mathrm{GeV}^{2}$ and a mass of the hadronic system $W_{X}>50 \mathrm{GeV}$. A LO calculation and alternatively the PYTHIA generator for the simulation of photons radiated by the quark $(Q Q)$ together with photons radiated from the electron $(L L)$ as predicted by RAPGAP describe the shape of the $E_{T}^{\gamma}$ and $\eta^{\gamma}$ distributions reasonably well, but lie below the data by $44 \%$ (LO) and $48 \%$ (MC). Both, 
Photon plus no-jets

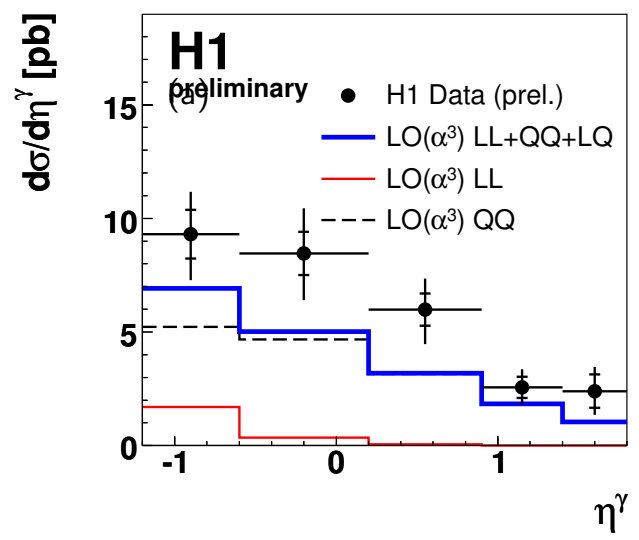

Photon plus jets

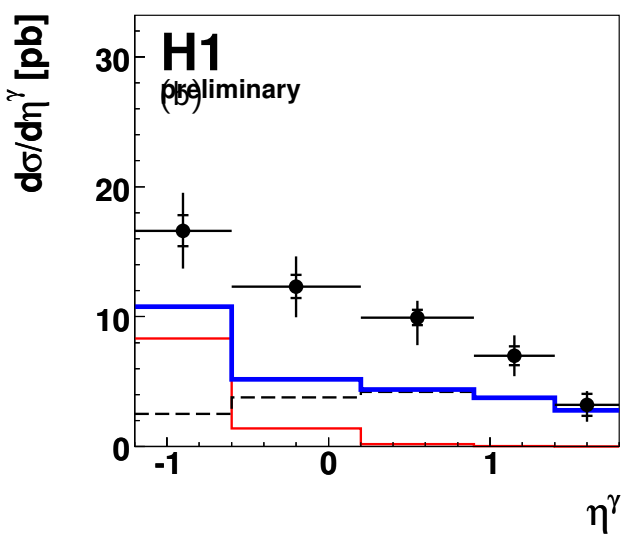

Figure 2: Differential cross sections $d \sigma / d \eta^{\gamma}$ for photon plus no-jets (a) and photon plus jets (b), with $P_{T}^{j e t}>2.5 \mathrm{GeV}$ and $-1.0<\eta^{\text {jet }}<2.1$. The cross sections are compared to a leading order, $\mathcal{O}\left(\alpha^{3} \alpha_{s}^{0}\right)$ as in Figure 1.

the LO calculation and the MC predictions are most significantly below the data at low $Q^{2}$. The cross sections for events with no or at least one hadronic jet are underestimated by the LO and MC predictions by a similar factor as in the inclusive measurement. Again the expectations are furthest below the data at low $Q^{2}$. The shapes of $d \sigma / d E_{T}^{\gamma}$ and $d \sigma / d \eta^{\gamma}$ are described reasonably well.

\section{References}

[1] Slides:

http: //indico . cern. ch/contributionDisplay . py? contribId=215\&sessionId=6\&conf Id $=9499$

[2] J. Breitweg et al. [ZEUS Collaboration], Phys. Lett. B 472 (2000) 175

[3] A. Aktas et al. [H1 Collaboration], Eur. Phys. J. C 38 (2005) 437

[4] S. Chekanov et al. [ZEUS Collaboration], Eur. Phys. J. C 49 (2007) 511

[5] S. Chekanov et al. [ZEUS Collaboration], Phys. Lett. B 595 (2004) 86

[6] A. Gehrmann-De Ridder, T. Gehrmann and E. Poulsen, Phys. Rev. Lett. 96 (2006) 132002

[7] A. Gehrmann-De Ridder, T. Gehrmann and E. Poulsen, Eur. Phys. J. C 47 (2006) 395

[8] T. Sjöstrand et al., PYTHIA 6.2 Physics and Manual (Version 6.224 is used)

[9] H. Jung, Comput. Phys. Commun. 86 (1995) 147 (Version 3.1 is used).

[10] I. Abt et al., Nucl. Instr. and Meth. A 386 (1997) 310, ibid, 348.

[11] R. D. Appuhn et al., Nucl. Instrum. Meth. A 386 (1997) 397.

[12] B. Andrieu et al. [H1 Calorimeter Group], Nucl. Instrum. Meth. A 350 (1994) 57.

[13] B. Andrieu et al. [H1 Calorimeter Group], Nucl. Instrum. Meth. A 336 (1993) 499.

[14] S. D. Ellis and D. E. Soper, Phys. Rev. D 48 (1993) 3160 